\title{
A General Parsing Algorithm with Context Matching for Context-Sensitive Graph Grammars
}

\author{
Yang Zou, Xiaoqin Zeng, Yun Zhu \\ Institute of Intelligence Science and Technology \\ School of Computer and Information, Hohai University \\ Nanjing, China \\ \{yzou, xzeng\}@hhu.edu.cn, zhuyunhhu@163.com
}

\author{
Tingting Sha \\ 5G Innovation Center \\ China United Network Communications Group Co., Ltd. \\ Beijing, China \\ shatt@ chinaunicom.cn
}

\begin{abstract}
Context-sensitive graph grammars have been intuitive and rigorous formalisms for specifying visual programming languages, as they are sufficient expressive and equipped with parsing mechanisms. Parsing has been a fundamental issue in the research of context-sensitive graph grammars. However, the existent parsing algorithms are either inefficient or confined to a minority of graph grammars. This paper proposes a general parsing algorithm with two embedded strategies, one is context matching, and the other is production set partitioning. The two strategies can greatly narrow down the search space of redexes and thus considerably improve the parsing performance, even though the worst-case time complexity is not theoretically reduced. Moreover, a case study along with detailed analysis is provided to demonstrate the paring process and parsing performance of the proposed algorithm.
\end{abstract}

Keywords-visual languages; context-sensitive graph grammar; context matching; production set partitioning; parsing algorithm

\section{INTRODUCTION}

Visual Programming Languages (VPLs) have been widely and frequently adopted in many disciplines of computer science. Like the textual programming languages that are usually equipped with proper formal syntax definition and parser, VPLs also need the support from such mechanisms. Quite a few approaches have been proposed for the specification and parsing of VPLs [1-3]. As a natural extension of formal grammar theory, graph grammars offer the mechanisms that can formally specify and parse VPLs [4], just like formal grammars do for string languages. In contrast to formal grammars, graph grammars consist of a set of productions in form of a pair of graphs rather than strings. However, the generalization from formal grammars to graph grammars brings about a new problem, the embedding problem [5].

Various graph grammar formalisms have been proposed in the literature [6-9], most of which are context-free or contextsensitive. The expressive power of a graph grammar depends on the type it belongs to as well as the embedding mechanism being integrated into the productions [10]. Context-sensitive graph grammars tend to be more expressive than context-free ones, when identically confined to less complex embedding mechanisms and invariant embedding in particular. Since context-free graph grammars even have difficulty in specifying a large portion of graphical VPLs [8-9], recent research in this discipline pays much more attention to context-sensitive graph grammars [11-13].

The existent context-sensitive graph grammar formalisms include Layered Graph Grammar (LGG) [8], Reserved Graph Grammar (RGG) [9], Contextual Layered Graph Grammar (CLGG) [14], Spatial Graph Grammars (SGG) [15], ContextAttributed Graph grammar (CAGG) [16], Edge-based Graph Grammar (EGG) [17], Temporal Graph Grammar (TGG) [13], etc. LGG and RGG are representative formalisms among them. CLGG, SGG, and TGG are typical extensions of LGG, RGG, and EGG, respectively. CLGG supports three extra mechanisms in productions to define more complex VPLs, SGG extends RGG by adding to productions a kind of spatial specification mechanism, and TGG generalizes LGG by augmenting temporal specification ability to productions.

In context-sensitive graph grammars, contexts pertaining to a production generally refer to the subgraphs neighboring to the rewritten portion of its left graph in potential host graphs [5], which describe the situations under which the production can be applied. According to how the context portion of a production is expressed, these formalisms are divided into two categories: explicit and implicit [18]. LGG and RGG are the typical examples of the former and the latter, respectively. An inherent weakness of implicit formalisms is that they are not intuitive. This is due to the fact that the context portion of a production is even not the complete immediate contexts. For example, the context portion of RGG productions is a set of marked vertices whose exact meaning is undefined. To make implicit context in productions explicit, a formal definition of context is proposed and the properties are characterized in [18], which provide a theoretical foundation for the computation of context.

Parsing has been one of the fundamental issues in the study of graph grammars. The LGG formalism is equipped with a parsing algorithm that consists of a top-down phrase for searching potential redexes and a bottom-up phrase for derivation from the initial graph to a given host graph [8]. Obviously, the process is rather complicated. RGG provides a naive Selection-Free Parsing Algorithm with polynomial time complexity, provided that the graph grammars are selection-free [9]. The condition selection-free is similar to the concept of local confluence in the literature. Unfortunately, this condition is not frequently satisfied in applications [18]. To deal with this problem, a general parsing algorithm is described in [19].

DOI reference number: 10.18293/DMSVIVA20-008 
Without a doubt, the mechanism of backtracking will inevitably appear in the parsing process, so as to traverse the search space that exponentially increases with the size of the host graph. Therefore, how to reduce backtracking becomes a critical challenge in parsing algorithms.

As to reducing backtracking, which is commonly caused by blind trials, i.e., reduction steps with unnecessary redexes, a feasible way is to try to avoid as many as possible irrelevant productions and unnecessary redexes in each step of reduction, namely to push less redexes into the stack in that they will probably be popped out for reduction in backtracking at a later time during the parsing process. Context can be employed to serve this purpose. When a redex is found in the host graph, the contexts of the corresponding production can be used to further check whether it is valid by matching the contexts with its circumstance in the host graph. If it is not matched, the redex can be directly discarded. Consequently, the potential backtracking is reduced. The approach to computation of context is proposed in [20], paving the way for context applications.

The technical contributions in this paper are as follows: Based on the RGG formalism, it proposes a general parsing algorithm with two essential strategies embedded, one is context matching, and the other is production set partitioning. The former can be utilized to reexamine the found redexes to exclude the unnecessary redexes, whereas the latter can be employed to precisely choose relevant instead of all the productions so as to narrow down the search for redexes. The two strategies will considerably improve the parsing performance of the proposed algorithm, even though the worst-case time complexity is not theoretically reduced. Moreover, a case study along with detailed analysis is provided to illustrate the paring process and parsing performance of the algorithm.

The remaining of the paper is organized as follows: Section 2 reviews the RGG formalism, and the notions of partial precedence graph and context. Section 3 presents a general parsing algorithm with two strategies established on the notions. Section 4 conducts an analysis of the algorithm from two aspects. Finally, Section 5 concludes the paper and proposes future research.

\section{PRELIMINARIES}

\section{A. The RGG Formalism}

A graph grammar consists of an initial graph and a set of productions (also called graph rewriting rules). Each production consists of two graphs called left graph and right graph respectively, and can be applied to another graph called host graph. Each node in a production is either a terminal or a nonterminal node. A graph grammar defines a graph language composed of those graphs that can be derived from the initial graph by repeated applications of the productions and whose nodes are all terminal ones.

In this paper, RGG is taken as the representative of implicit context-sensitive graph grammar formalism. RGG is a contextsensitive graph grammar formalism [9]. It introduces a nodeedge format to represent graphs in which each node is organized as a two-level structure, where the large surrounding rectangle is the first level, called super vertex, and other embedded small rectangles are the second level, called vertices. Either a vertex or a super vertex can be the connecting point of an edge. In addition to the two-level node structure, the RGG also introduces a marking technique that divides vertices into two categories: marked and unmarked ones. Each marked vertex of a production is identified by an integer that is unique in the left or right graph where the vertex lies. A production is properly marked if each marked vertex in the left graph has a counterpart marked by the same integer in the right graph, and vice versa. In the process of a production application, when a redex is matched in a host graph, each vertex that corresponds to a marked vertex in the left or right graph preserves its associated edges connected to nodes outside of the redex, which avoids the appearance of dangling edges during the subsequent subgraph replacement provided that an additional embedding rule is also enforced. The embedding rule states that if a vertex in the right (or left) graph of a production is unmarked and has an isomorphic vertex in the redex of a host graph, then all the edges connected to the vertex should be completely inside the redex.

An RGG that specifies process flow diagrams is depicted in Figure 1, and a simple process flow diagram is shown in Figure 5(a).
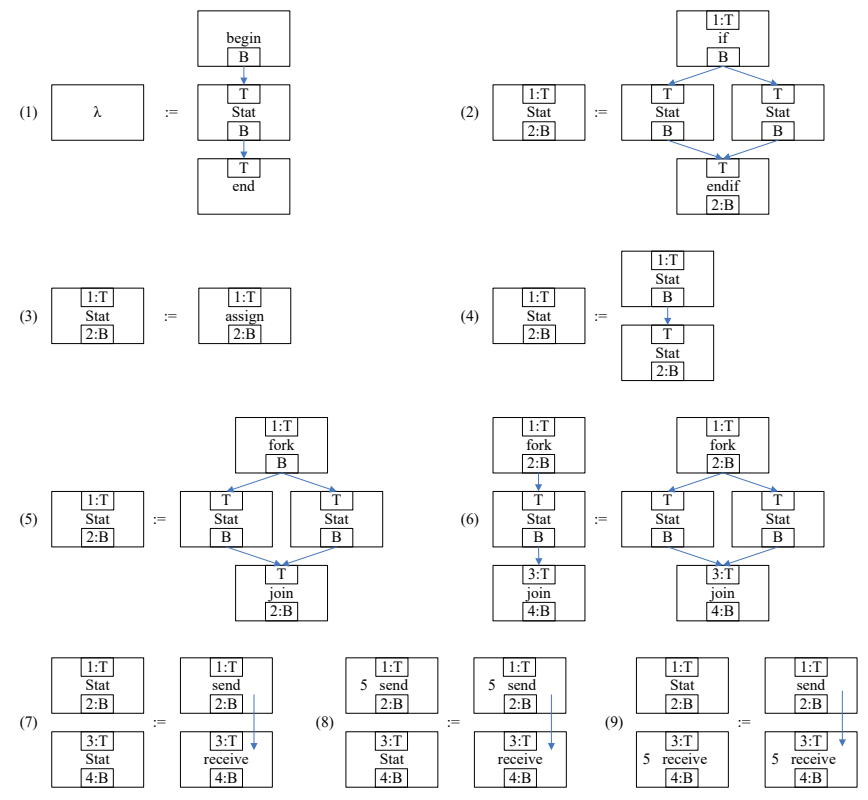

Figure 1. A graph grammar for process flow diagrams.

\section{B. Basic Concepts and Notations}

For the sake of clarity and simplicity, some basic concepts and notations are listed below. Note that graphs are directed ones in the node-edge format and only vertices in productions might be marked.

$R G G$ : A reserved graph grammar is a triple $(A, P, \Omega)$, where $A$ is an initial graph, $P$ a set of graph grammar productions, $\Omega$ a finite label set consisting of two disjoint sets $\Omega^{T}$ and $\Omega^{N T}$ (called terminal label set and nonterminal label set, respectively). For any production $p:=(L, R) \in P$, three conditions are satisfied: $R$ is non-empty, $L$ and $R$ are both over $\Omega$, and the size of $R$ are not less than that of $L$. 
$p:=(L, R):$ A production with a pair of marked graphs: the left graph $L$ and right graph $R$. The notations $p . L$ and $p . R$ represent the left and right graph of a production $p$, respectively. For any graph $G, G . N$ and $G$.E denote the set of nodes and edges, respectively; for any edge $e, s(e)$ and $t(e)$ represent the source and target vertex of $e$, respectively, and $l(e)$ is the label on $e$.

The function $M c c$ is a mapping from graphs to their sets of maximally connected subgraphs (components).

Redex: A redex is a subgraph in a host graph that is isomorphic to the left or right graph of a production.

A production's L-application to a host graph is to find in the host graph a redex of the left graph of the production and replace the redex in the host graph with the right graph of the production, while an R-application is a reverse process, i.e., to find a redex of the right graph of the production and replace the redex with the left graph.

Direct Partial Precedence: Let $g g:=(A, P, \Omega)$ be an RGG, and $p_{1}, p_{2} \in P$ be two productions, $p_{1}$ directly partially precedes $p_{2}$, denoted as $p_{1} \preccurlyeq_{d} p_{2}$, if $\exists S \subseteq M c c\left(p_{2} . L\right)$ such that $S \subseteq M c c\left(p_{1} . R\right)$. The direct partial precedence relation between them is denoted by the pair $\left\langle p_{1}, p_{2}\right\rangle$. The direct partial precedence relation on the set $P$ of productions is defined as $\preccurlyeq_{P}=\left\{\left\langle p_{1}, p_{2}\right\rangle \mid p_{1}, p_{2} \in P \wedge p_{1} \preccurlyeq_{d} p_{2}\right\}$.

The partial precedence relation is the closure of the direct partial precedence relation on a set $P$. Partial precedence is a kind of relation between a pair of components chosen from two distinct productions, whereas total precedence describes the same relation between two sets of components from a subset of productions and a single production respectively.

\section{Partial Precedence Graph}

The notion of partial precedence graph is proposed in [21], which is excerpted as follows:

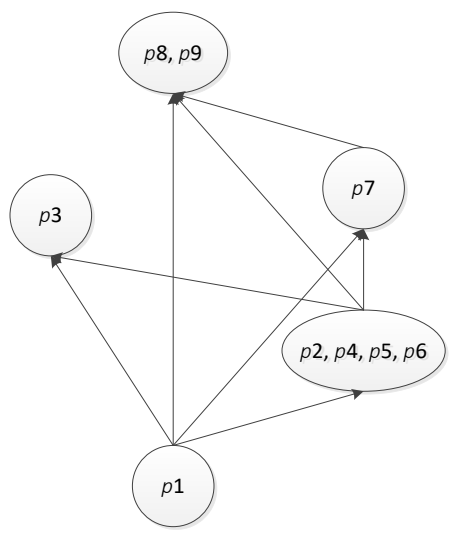

Figure 2. The partial precedence graph of the production set of an RGG.

Definition 1. Let $P$ be the production set of an RGG and $\preccurlyeq_{P}$ the direct partial precedence relation on $P$. A subset $D \subseteq P$ is a precedence ring if $\forall p, p^{\prime} \in D\left(p \neq p^{\prime} \rightarrow p \leqslant p^{\prime} \wedge p^{\prime} \leqslant p\right)$. A precedence ring $D$ is maximal if there does not exist another precedence ring $D^{\prime} \subseteq P$ such that $D \subset D^{\prime}$.
Definition 2. Let $P$ be the production set of an RGG, $\preccurlyeq_{P}$ the direct partial precedence relation on $P$, and $W=\left\{D_{1}, \cdots, D_{k}\right\}$ the set of maximal precedence rings of $P$. The partial precedence graph of $P$ is the pair $(W, E)$, where $E=\left\{\left(D_{i}, D_{j}\right) \mid \exists p, p^{\prime} \in\right.$ $\left.P\left(p \in D_{i} \wedge p^{\prime} \in D_{j} \wedge i \neq j \wedge p \preccurlyeq_{d} p^{\prime}\right), 1 \leq i, j \leq k\right\}$ is the set of edges between the elements of $P r$.

The partial precedence graph of the production set of the RGG in Figure 1 is depicted in Figure 2, where each node is a maximal precedence ring that is represented as a cycle or an eclipse, depending on the number of productions it contains. The node including only the first production $p 1$ can be neglected. A conclusion with respect to the partial precedence graph is drawn in [21] that if a host graph can be parsed by a graph grammar, then a valid reduction that conforms to the partial precedence graph of the graph grammar exists.

\section{Notion of Context}

The sets of partial or total precedence relations with respect to a graph grammar establish an order of production applications, which can be exploited to discover potential situations in which any of the productions is applicable for derivation. The situations are referred to as contexts.

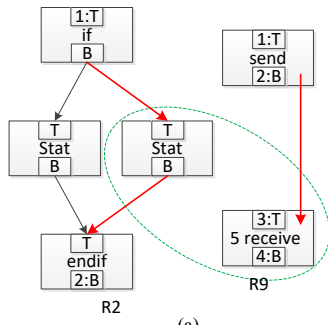

(a)

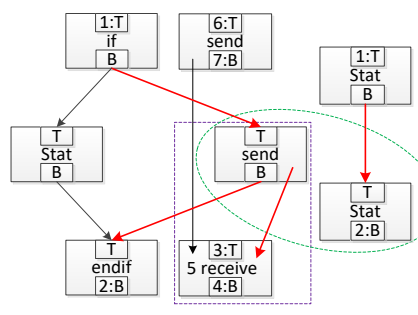

(b)
Figure 3. Contexts. (a) A level-1 context of $p 9$. (b) A level-2 context of $p 8$.

Given two productions $p_{1}$ and $p_{2}$, if $p_{1}$ directly partially precedes $p_{2}$, then $p_{1} \cdot R$ contains a context of $p_{2}$ or merely a portion of a context, depending on whether $p_{2} . L$ consists of only one or at least two maximally connected components. As for the former case, $\left\{p_{1}\right\} \prec_{d} p_{2}$ readily holds and a context of $p_{2}$ immediately follows; whereas in the latter case, a subset of productions involving $p_{1}$ that directly totally precedes $p_{2}$ is pursued so as to form a complete context for $p_{2}$. As a third case, a total precedence relation can be sought to build a rather deeper complete context. A formal definition on context can be found in $[20]$.

A context is of the form $(U, Z)$, where $U$ is the context subgraph, and $Z$ is the connection between the context and the redex.

Two contexts at different levels of a production are shown in Figure 3. A level-1 context of $p 9$ is shown in Figure 3(a), where the left component of the graph is $R 2$ (the right graph of production 2), the right one is $R 9$, the subgraph enclosed by the green dashed ellipse is the redex of $L 9$ (the left graph of $p 9$ ), and the context consists of two parts $U$ and $Z: U$ is the rest of the whole graph minus the redex and $Z$ the set of thick red edges that connect $U$ to the redex. Figure 3(b) shows a level-2 context of $p 8$. In this graph, the left and right component is $R 10$ (an 
extended production of $p 9$ with context) and $R 4$, respectively, the subgraph enclosed by the purple dashed rectangle is the isomorphic image of the underlying production of $p 10$, and the one surrounded by the green dashed eclipse is the redex of the left graph of $p 8$.

\section{GENERAl PARSING AlgorithM}

In this section, two algorithms that correspond to two parsing strategies: production set partitioning and context matching, are presented, and then a general parsing algorithm with the two strategies embedded follows.

\section{A. The Strategy of Context Matching}

Apparently, backtracking is the main cause of high time complexity of a general parsing algorithm, due to its blind trial of reductions. During the process of parsing a host graph, some unnecessary redexes are found and the corresponding reduction steps are conducted, and consequently a final graph will be obtained that is not the initial graph of the involved graph grammar and to which no more reductions can be conducted. This gives rise to backtracking in the process of parsing. Therefore, identifying unnecessary redexes so as to avoid unnecessary reductions is a direct and effective way to improve parsing performance. In this paper, the unnecessary redexes are termed as false positive redexes.

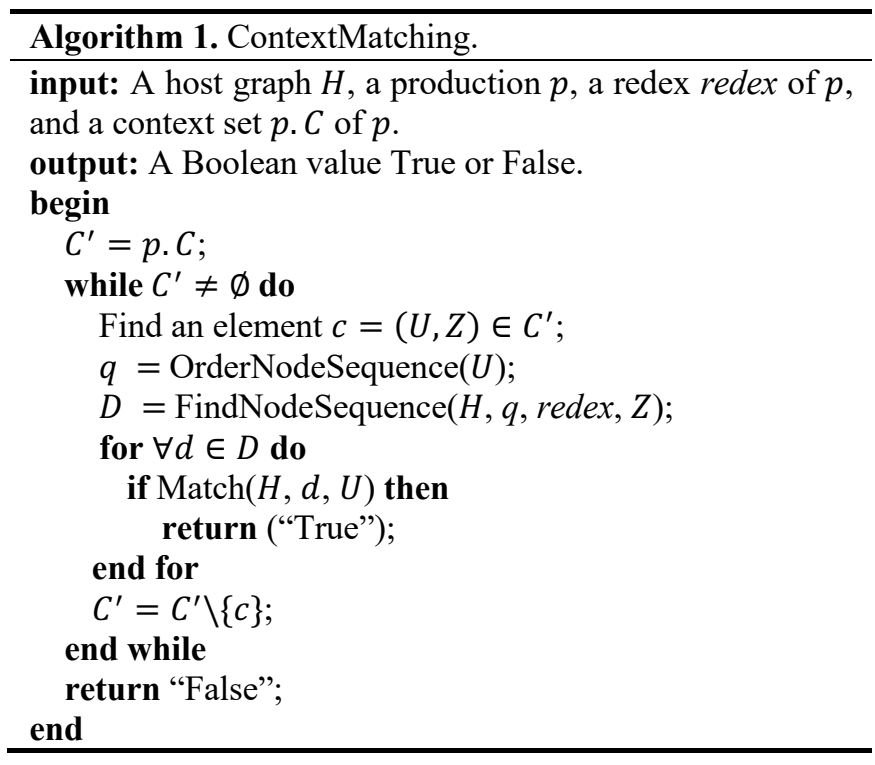

As for the RGG formalism, a subgraph will be found as a redex of a production's right graph in a host graph only when it is isomorphic to the right graph and the associated constraint on unmarked vertices is satisfied, regardless of the circumstance in which the subgraph lies. The circumstance, also called situation, refers to the rest of the host graph minus the written portion of the redex.

A strategy called context matching can be adopted to exclude false positive redexes by using the information of its situation. When a redex is found, an additional test is conducted to match the situation with contexts of the corresponding production.
Note that contexts of a production are potential situations in which it can be applied for derivation, and conversely, for reduction. Theoretically, the situation of a redex must match a context of any level (if applicable) for the corresponding production. The result of context matching is employed to determine whether a redex is false positive or true positive.

A redex is defined as true positive if the situation is matched with a level-1 context of the production to which it corresponds, or false positive, otherwise. If a redex is examined as false positive, it will be directly discarded, i.e., excluded from the set of candidates for reduction.

The procedure OrderNodeSequence $(U)$ sequences the nodes of the graph $U$ in terms of the alphabetic order of their labels; the procedure FindNodeSequenceSet $(H, q$, redex, $Z$ ) returns all the possible node sequences from the host graph $H$, each of which is the same as the given node sequence $q$ such that the set $Z$ exactly specifies the connection between the redex and the nodes in $H$ that constitutes this node sequence; and the function $\operatorname{Match}(H, d, U)$ checks whether $U$ is isomorphic to a subgraph in the host graph $H$ whose node sequence is $d$, if so, "True" is returned.

\section{B. The Strategy of Production Set Partitioning}

Algorithm 2 computes the partition of a set of productions according to its partial precedence graph, and returns the result in the form of an array of sets.

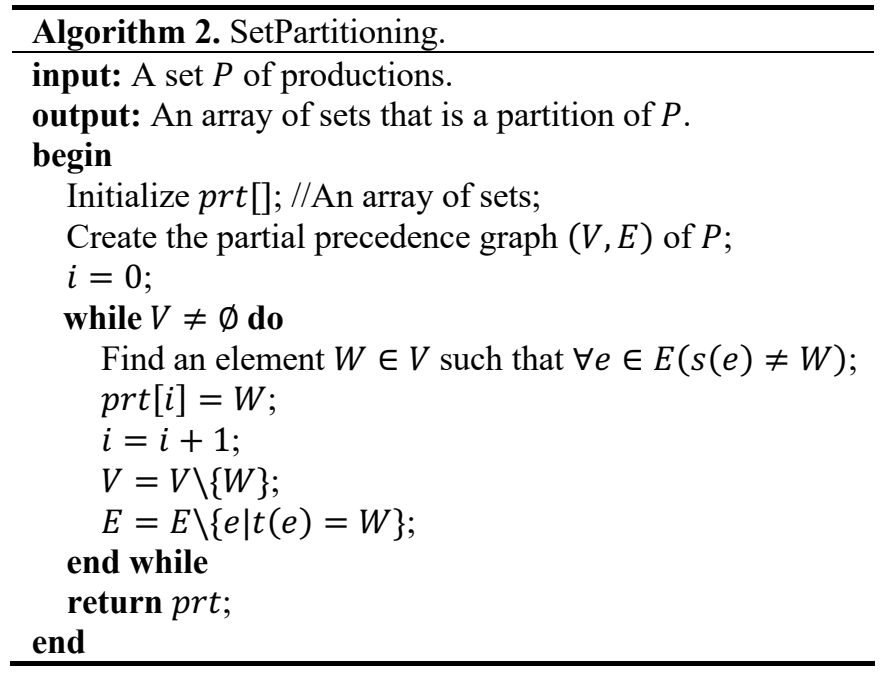

The function map: $P \rightarrow$ prt is a surjective mapping from a production set to its partition set (a set of disjoint subsets of $P$ whose union is $P$ ), where prt is the output of SetPartitioning $(P)$. That is, if $p \in P$, then $\operatorname{map}(p)=\operatorname{prt}[i]$, for some constant $i$ such that $p \in \operatorname{prt}[i]$. The function $\operatorname{succ}(Q)$ computes a successive subset in the partition, i.e., if $Q=\operatorname{prt}[i]$, $\operatorname{succ}(Q)=\operatorname{prt}[i+1]$, and if $Q$ is the last element, then $\operatorname{succ}(Q)$ is set to be an empty set.

\section{The General Parsing Algorithm}

Generally, parsing is such a process that attempts to perform a series of R-applications to reduce a given host graph to an 
initial graph with a designated graph grammar. It usually needs to deal with the following four interrelated steps:

- Search for the redexes of a production's right graph in a host graph;

- conduct an R-application with a certain redex to produce a new intermediate graph from the initial host graph or current intermediate graph;

- apply in turn the above two steps repeatedly, trace all the reduction paths, and backtrack if no more reductions can be made to the current intermediate graph that is not the initial graph;

- halt until a path leading to the initial graph is found or all possible paths have been exhausted.

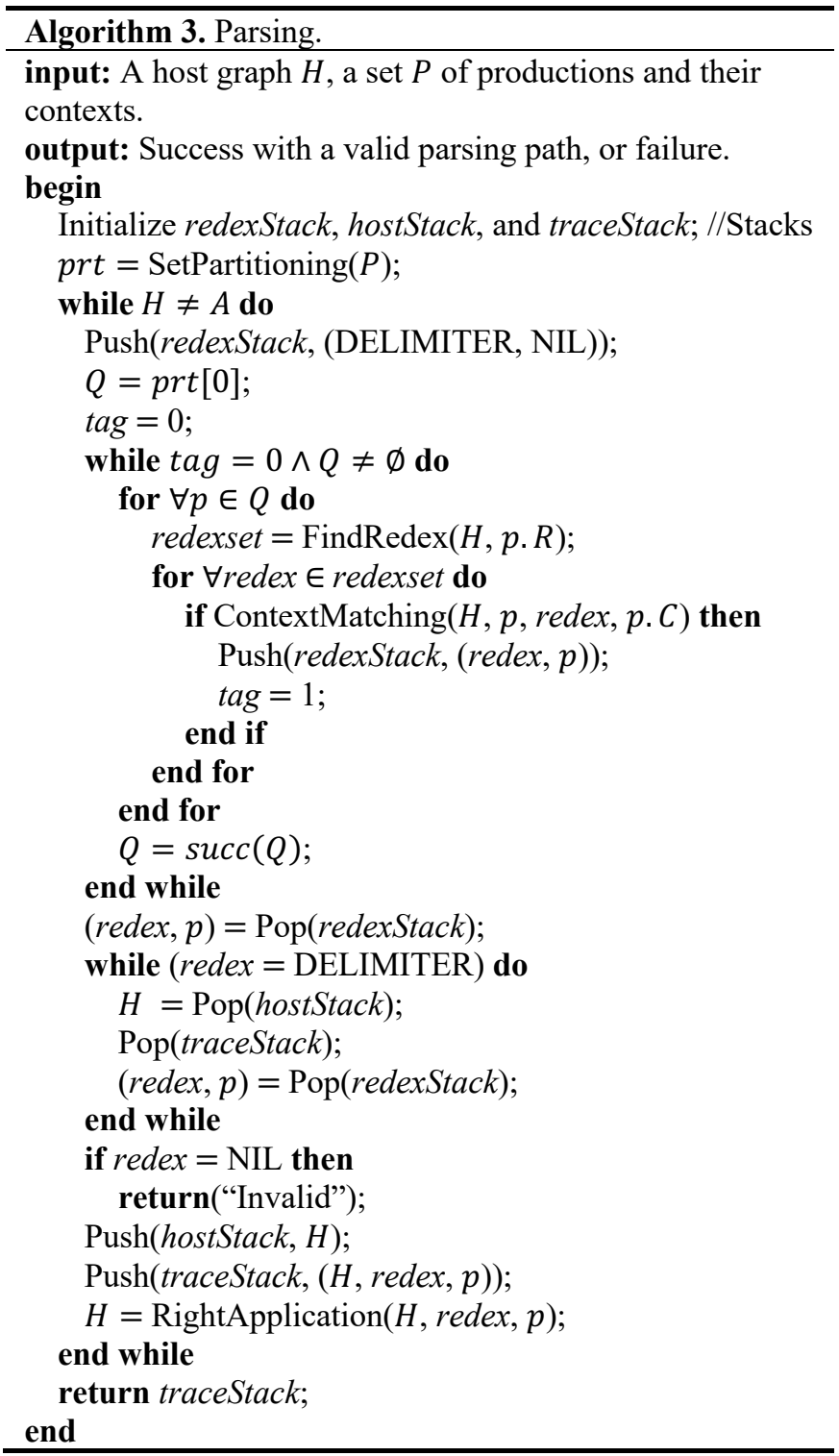

Algorithm 3 is a general parsing algorithm. It tries to trace all possible reduction paths starting from a given host graph to decide whether there exists one path that leads to the initial graph.
An assumption is that two basic procedures have already been available, one is searching for all the redexes of a production's right graph and the other is the accomplishment of an R-application. Further details on these two procedures is not discussed, since the procedures with similar functions can be found in the literature [1]. Three stacks are employed to record the redexes found, the intermediate host graph yielded, and the successful reduction path returned, respectively. As there is a need in the tracing to maintain a correspondence between a redex and its host graph for performing a reduction and this correspondence is usually many to one, it introduces a delimiter in the redex stack to delimit a group of redexes that corresponds to the same host graph. The delimiter makes the correspondence manageable by synchronizing the contents in the two stacks redexStack and hostStack. The algorithm takes a host graph and a set of productions and their contexts as input parameters and returns a definite answer to indicate whether the graph is valid or not. When the graph is valid, a successful reduction path is returned accordingly.

Two essential strategies are embedded in the general parsing process.

- Production set partitioning. The set of productions of a given graph grammar are partitioned into several disjoint subsets in terms of its partial precedence graph. Meanwhile, the subsets are ordered in accordance to the partial order suggested by the graph. When searching for redexes in an intermediate host graph, only a subset rather than the whole set of productions will be traversed. This operation is done by Algorithm 2.

- Context matching. Upon all the redexes of a production's right graph in an intermediate host graph are computed, the algorithm will not directly push them one by one into the stack redexStack in preparation for possible backtracking. Instead, it further decides whether each of them is an "appropriate" redex by matching the circumstance in the host graph where it is situated with its contexts. If a redex is verified in this way, it will then be pushed into the stack; otherwise, it will be discarded. This operation is done by Algorithm 1.

Some details of the algorithm are explained as follows. In the outmost while-loop, $Q$ is an element from the partition set prt, denoting a subset of productions whose redexes will be searched in H. tag is Boolean value, indicating whether there is a redex of some production in $Q$ that has been pushed into the redexStack. If so, $\operatorname{tag}$ is set to 1 ; otherwise, $\operatorname{tag}$ remains 0 and $Q$ will be replaced by its successor. Noticeably, the first embedded whileloop collects merely the redexes of the productions in $Q$ whose contexts have been matched, rather than all the redexes of $P$. The second while-loop conducts backtracking when no redexes are found in the intermediate host graph $H$ during the first whileloop. traceStack is employed to record the parsing trace.

\section{AlgORITHM ANALYSIS}

In this section, we first analyze the time complexity of Algorithm 3, then present a case study to illustrate how the algorithm improves parsing performance, and finally summarize its characteristics and indicate the situations in which it can perform efficiently. 


\section{A. Time Complexity}

Theorem 1. The worst-case time complexity of Algorithm 3 is $O\left((m / r !)^{h}\left(h ! h^{h}\right)^{r}\right)$, where $h$ is the number of nodes in the host graph to be parsed, $m$ is the number of productions of the graph grammar, $n$ is the maximal number of components in the left or right graphs of the productions, and $r$ is the maximum number of nodes in the right graphs of the productions.

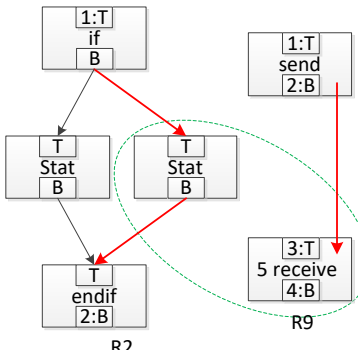

(a)

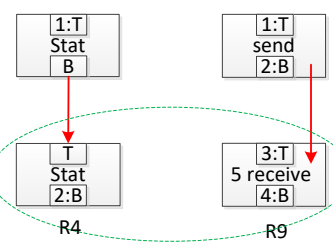

(c)

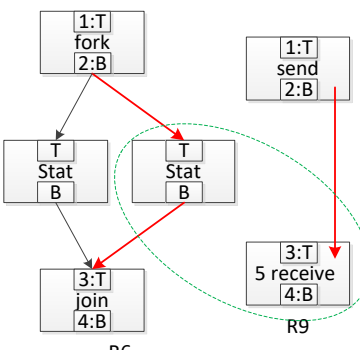

(e)

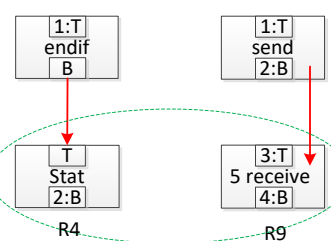

(g)

Figure 4. Some contexts of production $p 9$.

Proof. An assumption is that the maximal degree of all the nodes in a host graph is a constant. In Algorithm 2, the creation of the partial precedence graph accounts for the time taken, that is $O\left(m^{2}\left(m+n^{2} r^{2}\right)\right)$ [21], and so is the time complexity of the algorithm. But, from the perspective of Algorithm 3, Algorithm 2 takes $O(1)$, as $m, n$, and $r$ are small constants for a graph grammar.

Similarly, the time complexity of a call for Algorithm 1 in Algorithm 3 is also $O(1)$, since the number of contexts for a production used for this purpose is a constant, and the time taken by the procedure FindNodeSequenceSet $(H, q$, redex, $Z)$ is merely dependent on the degree of the nodes directly connected to the redex through $Z$, but has nothing to do with the cardinality of $H$.

The underlying structure of Algorithm 3 is similar to the algorithm described in [19]. In the worst case, calls for Algorithm 1 and Algorithm 2 may not essentially reduce the search space of productions and redexes. Hence, the time complexity of Algorithm 3 is also $O\left((m / r !)^{h}\left(h ! h^{h}\right)^{r}\right)$.

\section{B. A Case Study}

we introduce a typical example to illustrate how the proposed algorithm reduces the search space during the parsing process.

Figure 4 lists some typical examples of the level-1 contexts for the production $p 9$. The left graph of $p 9$ is composed of two components, one is the node "Stat", and the other is "receive". The former component appears 5 times in the right graphs of four productions, and the latter occurs 3 times in the right graphs of three productions. Therefore, the level-1 contexts of $p 9$ totally is 15 , i.e., the cardinality of the Cartesian product of the two constituents.

Figure 5 shows the deduction processes of a host graph parsed by the graph grammar depicted in Figure 1. The host graph is given in Figure 5(a). Graphs in (a)-(g) demonstrate a complete successful reduction, which can be generated by Algorithm 3. On the contrary, (h)-(j) present three intermediate host graphs that will lead to dead ends in next several deduction steps where backtracking have to be conducted.

In each graph of Figure 5, the subgraph surrounded by the dashed green rectangle (if exists) is a redex of some production's right graph, the bold red arcs connected to a redex are the contextual connection of the redex, and the subgraph surrounded by the dashed red rectangle (if exists) is the output of the Rapplication of a production to the redex in the anterior graph in a deduction.

Consider the case when a general parsing algorithm without the two strategies is adopted to parse the host graph in Figure $5(\mathrm{a})$.

Two redexes of $p 3$ and one redex of $p 9, p 7$ and $p 8$ can be found, which is shown in the dashed green rectangles. Ordinarily, five redexes will be pushed into redexStack, and then one will be popped out for deduction. We assume that the redex of $p 8$ is popped out first. Note that this enclosed subgraph is also the redex of both $p 9$ and $p 7$ simultaneously. After an R-application, one obtains an intermediate host graph, shown in (h), where the subgraph enclosed in the dashed red rectangle is the output of the R-application of $p 7$. In (h), only two redexes of $p 3$ exist, and twice R-applications of $p 3$ can be done accordingly, leading to a host graph that cannot be deduced any longer. As a matter of fact, other choices (except $p$ 9) lead to situations similar to (h), e.g., the graph (i). In these situations, backtracking is needed after a few reduction steps.

In contrast, Algorithm 1 tackles the host graph in Figure 5(a) in a quite different way. 


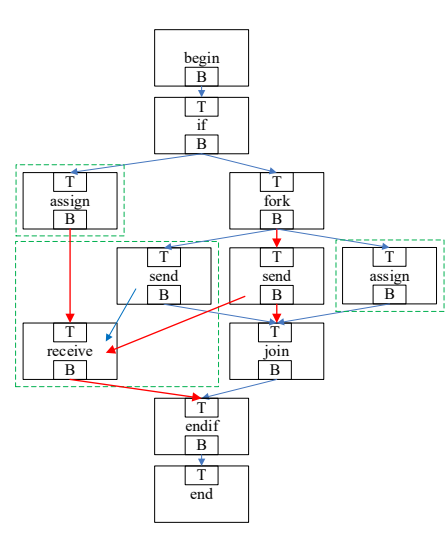

(a)

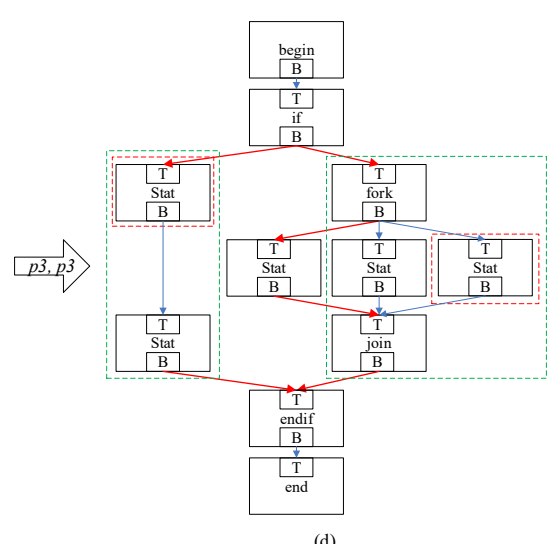

(d)

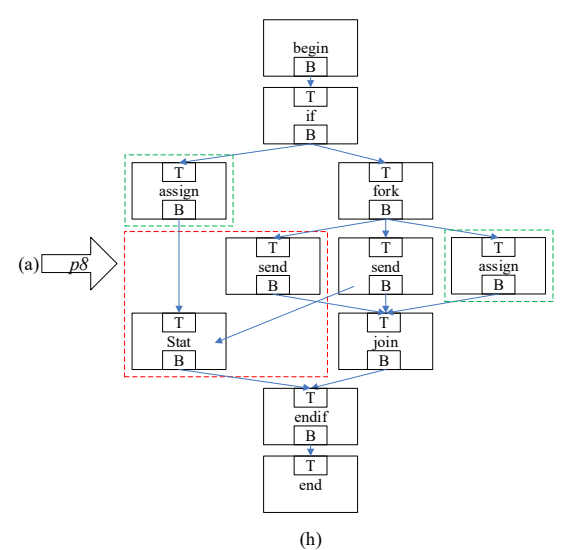

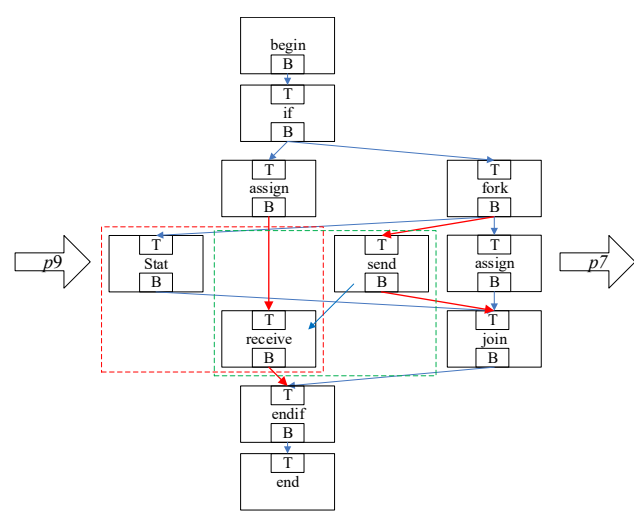

(b)

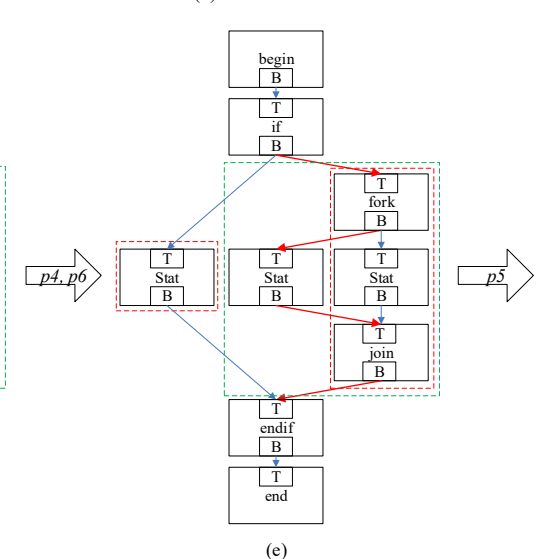

(e)

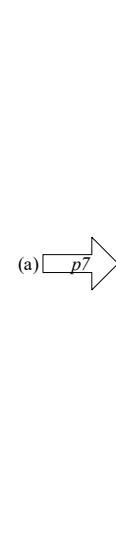

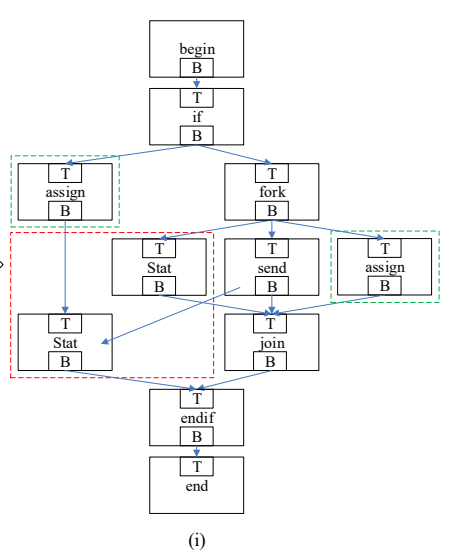

(i)

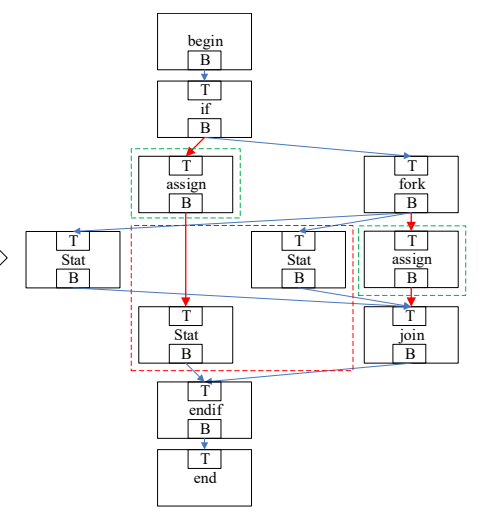

(c)

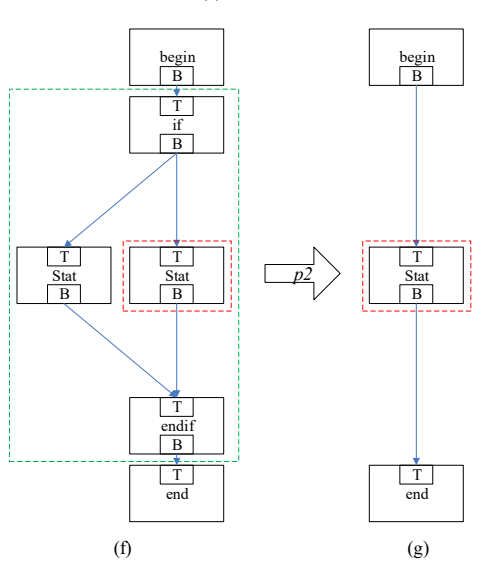

(g)

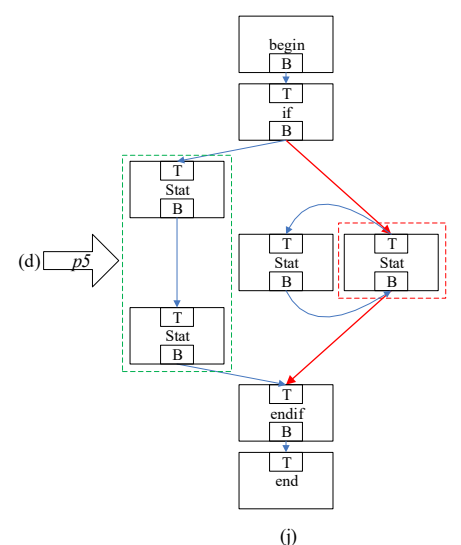

(j)

Figure 5. The parsing processes of a host graph. (a)-(g) A successful parsing path produced by Algorithm 3. (h)-(j) three intermediate host graphs where backtracking is necessary.

First, the set $P$ of productions are partitioned into 4 subsets: $\operatorname{prt}[0]=\{p 8, p 9\}, \operatorname{prt}[1]=\{p 7\}, \operatorname{prt}[2]=\{p 3\}, \operatorname{prt}[3]=$ $\{p 2, p 4, p 5, p 6\}$, and these subsets are sequentially ordered, in terms of the partial precedence graph of $P$. The redexes of productions from them will be handled in priority according to the order.

Then, in the first iteration of the outmost while-loop, only the redexes of productions in prt [0] are considered in this graph, that is, the redex of $p 8$ or $p 9$. Moreover, context matching can be utilized to further verify the redex found. Obviously, a context is matched for $p 9$, whereas no context is matched for $p 8$. A distinct character of the contexts for $p 9$ is that a node labeled "send" is the source node of an edge directed to the node "receive" in the redex. After the application of $p 9$ to the redex in (a), graph (b) is obtained.

Next, it enters the second iteration and repeats the above process. It searches graph (b) for redexes of the productions from prt [0] and finds the subgraph enclosed in the green rectangle, which is a redex of both $p 8$ and $p 9$. However, no contexts can be matched for both productions. Therefore, it tries the second subset $\operatorname{prt}[1]$ and obtains a redex of $p 7$. Similarly, context matching is conducted to the redex enclosed in the green 
rectangle. Then the redex is verified and a corresponding deduction step outputs (c).

In the third iteration, as there is no more redexes of productions from both prt[0] and prt[1] in (c), the only production in prt [2] is considered, which leads to (d).

The subgraph enclosed in the green dashed rectangle in (d) is a redex of both $p 5$ and $p 6$ from prt [3]. Once again, context matching is activated. A distinct character of the contexts for $p 6$ rather than for $p 5$ is that a node labeled "Stat" is simultaneously the target node of an edge originated from the node "fork" and the source node of an edge directed to the node "join" in the redex. Thus, $p 5$ is excluded and $p 6$ is selected for R-application, leading to (f). Finally, (g) follows. A valid deduction path is achieved even without backtracking. In the parsing process, only level-1 contexts are employed.

Otherwise, if $p 5$ is selected for deduction in the intermediate host graph (d), it then produces (j). An R-application of $p 4$ can be conducted to $(j)$, and the resulting graph is irreducible. In that situation, backtracking is inevitable.

\section{Discussion}

As is known, backtracking is the main cause for high time complexity of a general parsing algorithm. An ideal way is to avoid as many as possible unnecessary productions and redexes in every reduction step. The introduction of the two strategies is exactly for this purpose.

In each reduction step, not the whole set of productions of the graph grammar will be chosen for searching for redexes in a host graph. Instead, only a subset of it is selected by using the strategy of production set partitioning. For example, in the iterations of the first inner while-loop, only 1 or 2 out of the total 9 productions are picked for searching in most cases. Thus, this strategy can exclude a majority of unnecessary productions in each reduction step. The effectiveness of production set partitioning originates from locality principle for programs, as a graph grammar is essentially a program. Obviously, the more subsets a production set can be partitioned into, the more productions will be excluded by the strategy in reduction steps.

After being found, a redex of a production will be verified by context matching to check whether it is a true positive one. This is a critical means to reduce backtracking. For instance, in Figure 5(a), there is a subgraph that is a redex of $p 9, p 7$ and $p 8$; by means of context matching, the redex of $p 9$ is verified whereas other two redexes are denied; otherwise, the execution of R-applications of $p 8$ and $p 7$ to the host graph will produce the graphs in (h) and (i), respectively, any of which will arrive at situations of backtracking in a few more reduction steps. A similar situation arises in (d) where the subgraph enclosed by the right dashed green rectangle is a redex of both $p 6$ and $p 5$, and the strategy excludes the latter and avoids the situation depicted in (j) accordingly. In this example, context matching removes all the unnecessary redexes so as to eliminate backtracking. Hence, the strategy can exclude a certain number of unnecessary redexes in each reduction step. Clearly, when the right graphs of two productions share a common subgraph, their contexts are supposed to be distinct from each other, which is a premise to ensure the effectiveness of the strategy.
In summary, we believe that the introduced strategies can considerably improve the parsing performance of a general parsing algorithm for context-sensitive graph grammars.

\section{CONCLUSION AND FUTURE WORK}

Based on the RGG formalism, this paper proposes a general parsing algorithm for context-sensitive graph grammars. The algorithm is embedded with two essential strategies: one is context matching, and the other is production set partitioning. The former is utilized to reexamine the found redexes so as to exclude the unnecessary redexes, and the latter can be used to precisely choose the relevant productions to narrow down the searching space for redexes. Therefore, the two strategies can considerably improve the parsing performance, even though the worst-case time complexity is not theoretically reduced. Furthermore, a case study along with detailed analysis is provided to illustrate the paring process and parsing performance of the proposed algorithm.

Nevertheless, to what extent can the strategy of context matching reduce unnecessary redexes, and how is it related to the characteristics of contexts still need to be investigated. In our future work, feature analysis and integration for contexts will be conducted to facilitate the study on these issues.

\section{ACKNOWLEDGMENT}

This work is supported in part by the National Science Foundation of China under grant 61170089.

\section{REFERENCES}

[1] F.Ferrucci, G. Pacini, G. Satta, et al., "Symbol-relation grammars: a formalism for graphical languages," Information and Computation, 131(1), pp. 1-46, 1996.

[2] K. Marriott, "Constraint multiset grammars," IEEE Symposium on Visual Languages, St. Louis, Missouri, pp. 118-125, 1994.

[3] G. Costagliola, V. Deufemia, and G. Polese, "Visual language implementation through standard compiler-compiler techniques," Journal of Visual Languages and Computing, 18(2), pp. 165-226, 2007.

[4] G. Rozenberg (Ed.), Handbook on Graph Grammars and Computing by Graph Transformation, vol. 1: Foundations, World Scientific, 1997.

[5] J. L. Pfaltz, A. Rosefeld, "Web grammars," International Joint Conference on Artificial Intelligence, pp. 609-619, 1969.

[6] G. Engels, H. J. Kreowski, and G. Rozenberg (Eds.), Handbook of Graph Grammars and Computing by Graph Transformation, vol. 2: Applications, Languages, and Tools, World Scientific, 1999.

[7] H. Ehrig, H. J. Kreowski, U. Montanari, and G. Rozenberg (Eds.), Handbook of Graph Grammars and Computing by Graph Transformation, vol. 3: Concurrency, Parallelism, and Distribution, World Scientific, 1999.

[8] J. Rekers and A. Schürr, "Defining and parsing visual languages with layered graph grammars," Journal of Visual Languages and Computing, 8(1), pp. 27-55, 1997.

[9] D. Zhang, K. Zhang, and J. Cao, "A context-sensitive graph grammar formalism for the specification of visual languages," The Computer Journal, 44(3), pp. 187-200, 2001.

[10] M. Nagl, "Set theoretic approaches to graph grammars," International Workshop on Graph Grammars and Their Application to Computer Science, Lecture Notes in Computer Science, vol. 291, Springer Verlag, pp. 41-54, 1987.

[11] C. Zhao, J. Kong, and K. Zhang, Program behavior discovery and verification: a graph grammar approach, IEEE Transactions on Software Engineering, 36(3), pp. 431-448, 2010. 
[12] Y. Liu, X. Zeng, Y. Zou, and K. Zhang, "A graph grammar-based approach for graph layout," Software: Practice and Experience, 49(8), pp. $1523-1535,2018$.

[13] Z. Shi, X. Zeng, Y. Zou, et al., A temporal graph grammar formalism. Journal of Visual Languages and Computing, vol. 47, pp. 62-76, 2018.

[14] P. Bottoni, G. Taentzer, and A. Schürr, "Efficient parsing of visual languages based on critical pair analysis and contextual layered graph transformation," IEEE Symposium on Visual Languages, pp. 59-60, 2000.

[15] J. Kong, K. Zhang, and X. Zeng, "Spatial graph grammars for graphical user interfaces," ACM Transactions on Computer-Human Interaction, 13(2), pp. 268-307, 2006.

[16] Y. Zou, X. Zeng, and X. Han, "Context-attributed graph grammar framework for specifying visual languages," Journal of Southeast University (English Edition), 24(4), pp. 455-461, 2008.
[17] Y. Liu, Z. Shi, and Y. Wang, "An edge-based graph grammar formalism and its support system," International DMS Conference on Visualization and Visual Languages, pp.101-108, 2018.

[18] Y. Zou, J. Lü, and X. Tao, "Research on context of implicit contextsensitive graph grammars," Journal of Computer Languages, vol. 51, pp. 241-260, 2019. https://doi.org/10.1016/j.cola.2019.01.002

[19] X. Zeng, K. Zhang, J. Kong, and G. Song, RGG+: An enhancement to the reserved graph grammar formalism. In Proc. IEEE Symposium on Visual Languages and Human-Centric Computing, pp. 272-274, 2005.

[20] Y. Zou, X. Zeng, Y. Liu, and H. Liu, "Context computation for contextsensitive graph grammars: Algorithms and Complexities," Journal of Visual Language and Computing, vol. 1, pp. 15-28, 2019.

[21] Y. Zou, X. Zeng, Y. Liu, and H. Liu, "Partial precedence of contextsensitive graph grammars," International Symposium on Visual Information Communication and Interaction, pp. 16-23, 2017. 